\title{
RINGO: a novel ring polarimeter for rapid GRB followup
}

\author{
I. A. Steele ${ }^{a}$, S. D. Bates ${ }^{a}$, D. Carter $^{a}$, D. Clarke ${ }^{b}$, A. Gomboc ${ }^{a, c}$, C. Guidorzi ${ }^{a}$, A. Melandri $^{a}$,

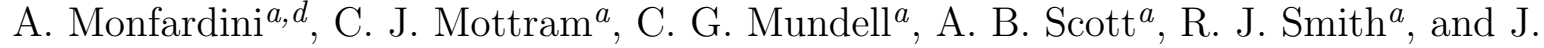 \\ Swindlehurst ${ }^{a}$ \\ ${ }^{a}$ Astrophysics Research Institute, Liverpool JMU, Twelve Quays House, Egerton Wharf, \\ Birkenhead CH41 1LD, UK; \\ ${ }^{b}$ Department of Physics \& Astronomy, The University, Glasgow, G12 8QQ, UK; \\ ${ }^{c}$ Faculty of Mathematics and Physics, University of Ljubljana, Jadranska 19, 1000 Ljubljana, \\ Slovenia; \\ ${ }^{d}$ ITC-IRST and INFN Trento, via Sommarive 18, 38050 Povo (TN), Italy
}

\begin{abstract}
We describe the design and construction of a novel optical ring-polarimeter (RINGO) for the Liverpool Telescope. The instrument is designed for rapid ( $<5$ minutes) followup observations of Gamma Ray Bursts in order to measure the early time polarization and its evolution for the first time. Sensitivity calculations and data reduction procedures are described, and the results of on-sky commissioning presented. The instrument is now on the telescope and in routine use during GRB followup.
\end{abstract}

Keywords: robotic telescopes, gamma ray bursts, polarimeters, astronomical instrumentation

\section{INTRODUCTION}

The Liverpool Telescope ${ }^{1}$ (LT) is a 2.0 metre fully robotic telescope. It specializes in time variable and rapid reaction astronomy. The telescope Acquisition \& Guidance (A\&G) unit can host up to five instruments ${ }^{2}$ with the beam able to be directed to one of four side ports via a folding mirror, or, with the folding mirror removed from the beam, to a straight through port.

A principle science goal of the Liverpool Telescope is the rapid ( $<5$ minutes) follow up of the optical transient sources associated with Gamma Ray Bursts (GRBs). ${ }^{3}$ This has had considerable success ${ }^{4}$ with multicolour lightcurves of bursts now routinely in production. ${ }^{5}$ An area that remains to be explored in early-time GRB studies however is polarimetry. There are good reasons to suspect that at very early times radiation from GRBs may be strongly polarized, and measurements of this would place strong constraints on their geometry and magnetic field strength and structure. With this in mind a novel optical polarimeter (RINGO) with the wide field and high time resolution necessary to observe GRBs has been fast-track developed and deployed on the Liverpool Telescope. In this paper we give a brief overview of the science drivers for the instrument, followed by details of its design and construction. We also discuss the data reduction procedure for the instrument, and give the results of our commissioning tests.

\section{SCIENCE DRIVER}

Despite the rapid development in our understanding of the physics of GRBs since the discovery of the first X-ray ${ }^{6}$ and optical ${ }^{7}$ afterglows in 1997, the fundamental emission mechanisms and early time evolution of the ejecta remain unknown. Given that GRBs lie at cosmological distances, they will remain spatially unresolved by all current and future instrumentation. Measurements of the degree and angle of polarization in their early-time light curves could therefore provide a uniquely powerful probe of the geometry of the ejecta and magnetic field strength and structure. Most importantly, the time evolution of the polarization properties provides the most stringent tests of current jet and internal shock model predictions. In turn, determination of the jet and magnetic field configurations is key for deriving the characteristics of the explosion, including its energetics. The diagnostic

email: ias@astro.livjm.ac.uk 


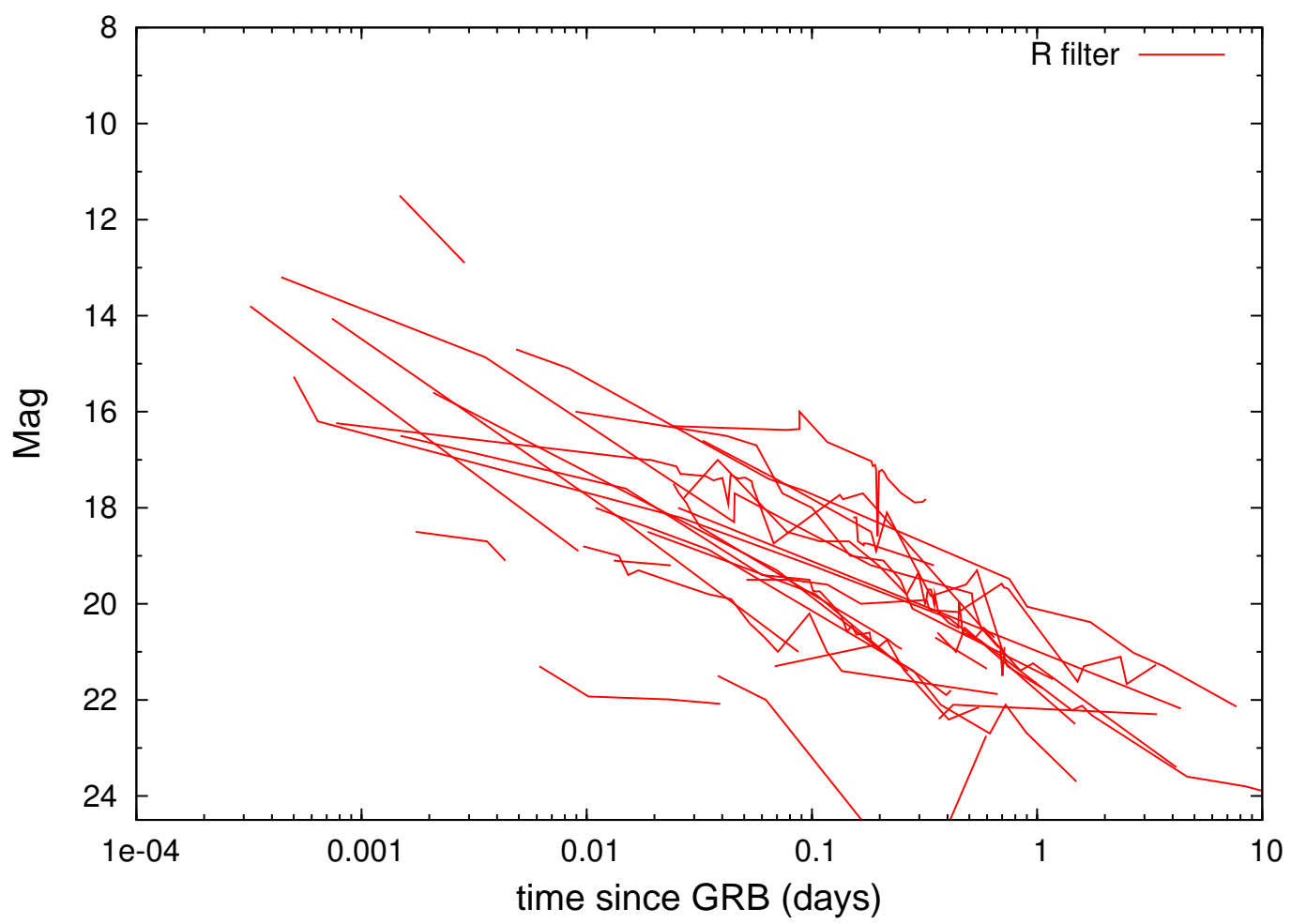

Figure 1. $R$ band early time lightcurves of GRB optical transients up to January 2006. The interval $0.001-0.01$ days corresponds to 1.5 - 15 minutes, this being the time window which RINGO is designed to exploit.

power of very early time polarization measurements is large and such measurements are most likely to lead to the next major observational breakthrough in GRB studies.

Long-duration GRBs are understood to be energetic explosions at cosmological distances, caused by the dissipation of a relativistic outflow, or fireball expansion, associated with the gravitational collapse of a massive star to a compact remnant. ${ }^{8}$ Although it is generally accepted that the afterglow emission is synchrotron radiation whose observed properties are broadly explained by a highly-relativistic geometrically-beamed fireball, the driving mechanism of the fireball and, in particular, the role of magnetic fields, is still unknown. It should also be noted that the emission mechanisms for prompt and afterglow optical sources could be quite different.

The first detection ${ }^{9,10}$ of polarization in a GRB afterglow (1.7\% in GRB 990510) and the corresponding achromatic break in its optical light curve provided support for beamed synchrotron and highlighted the importance of polarization studies of GRBs. Other late time measurements have also begun to be obtained. ${ }^{11}$ Whilst a critical first step in the study of polarization, these low-level detections occur many hours after the burst and therefore provide limited information on the physics and energetics of the initial explosion. In addition it is important to check if the polarization changes with time or whether temporal measurements provide a static value as a result of the interstellar medium along the line of site through the galaxy. Synchrotron radiation can be up to $60 \%-70 \%$ polarized, but observed values will be modified by the geometry and magnetic field structure of the emitting region and observer viewing angle. ${ }^{12}$

A number of complex fireball and jet models now exist and, although these are key for unifying observed $\gamma$ and X-ray properties of different classes of GRBs and X-ray flares, they are largely indistinguishable from one another on the basis of observed light curves alone. ${ }^{13}$ However, a collimated ultra-relativistic outflow produces a characteristic time evolution of the degree and position angle of polarization that depends on the ejection geometry and magnetic field structures. Models associated with the later afterglow phase and external shocks are now beginning to be constrained, although, to date, only three GRBs have useful late-time temporal coverage and, 
of these, only one GRB 020813 -has sufficient sampling to yield any firm conclusions. ${ }^{13}$ More importantly, our primary goal is to measure the polarization in the early emission phase when the magnetized and unmagnetized models differ most and internal shocks may dominate the emission. Indeed, at early and late times, polarization should be essentially zero in the absence of a magnetic field but this is substantially modified in the presence of a large-scale ordered magnetic field, driving the fireball expansion. Here the predicted polarization is high as $20 \%$ (not yet observed) in contrast to purely hydrodynamical models. ${ }^{14-16}$ We therefore conclude that polarimetry of the early-time optical emission may therefore be the most powerful diagnostic tool to investigate the fireball geometry, energetics, and dynamical evolution.

Overall the low levels of polarization measured to date in a handful of GRBs have required careful observational efforts on large telescopes due to lateness of the observations. Polarization sampling to date is insufficient to distinguish unambiguously between jet models. With the routine and rapid $\sim$ few arcminutes localization of GRBs with Swift, fast response facilities such as the Liverpool Telescope therefore have a unique opportunity to measure the polarization properties of GRBs at the earliest times, when the optical emission is expected to be brightest, the polarization properties are as yet unknown, and the models are most predictive.

\section{REQUIREMENTS}

\subsection{Polarimetric Accuracy and Sensitivity}

From the discussion of the science case presented above it can be seen that while the expected polarization may be as high as $\sim 20 \%$, it could also be of a similar magnitude to that which has been observed at late times $(\sim 1-2 \%)$. Therefore a target polarization accuracy of $0.1 \%$ was set. This will obviously be dependent on the object brightness, as polarimetry is in essence the comparison of two photometric measurements. A simple signal-to-noise calculation shows that for a 2.0 metre telescope such as the LT, the limiting photon-counting statistics imply that the $0.1 \%$ polarimetric accuracy can be obtained at magnitude $R \sim 15$ in a $\sim 10$ minute exposure. Examination of Figure 1 (note 0.001 days corresponds to $\sim 1.5$ minutes) shows that $\sim$ few $\%$ of the optical transients should be bright enough to have their early time polarization measured to that accuracy. A larger fraction $(\sim 10-20 \%)$ should have their polarization measurable to $\sim 1 \%$.

\subsection{Field of View}

Our experience with using the LT RATCam CCD Imager ${ }^{2}$ shows that its $4.6 \times 4.6$ arcminute field of view means that the OT (if any is present) falls on the CCD for over $90 \%$ of Swift BAT positions. ${ }^{3}$ In other words with such a field of view it is generally possible to find and identify optical transients purely from the Swift BAT trigger. This is preferable to waiting for a Swift XRT position as this can sometimes be delayed either by satellite pointing constraints or other reasons.

The LT A\&G box can host up to 5 instruments simultaneously, using a folding mirror to direct the beam to one of four side ports. The time to change between side ports is $\sim 30$ seconds. While in normal use this time is relatively unimportant, with a fast decaying (both in intensity and potential polarization signal) GRB optical transient, this delay can be significant. Given the expected rapid decay of the polarization signal, it was seen as vital that the polarization measurement be obtained as soon as possible after the burst trigger. Therefore the obvious strategy of locating the burst using the telescope CCD imager (RATCam) and then carrying out a reacquisition to place a narrow field polarimeter onto the burst was rejected as adding too much overhead (calculated at $\sim 2$ minutes including the exposure time for the CCD exposures) before making the polarimetric measurement. It was therefore decided that the field of view of the polarimeter should match that of the CCD imager (i.e. $>4 \times 4$ arcminutes).

We note here that in operation the plan is that a single RINGO observation is made at the start of every burst before the normal sequence of automated discovery images using the CCD imager is taken and analyzed in real time. If the automated pipeline ${ }^{3}$ then discovers a bright afterglow, then followup RINGO observations are then interspersed with normal CCD imaging for the next hour in order to measure the time evolution of the polarization signal. 


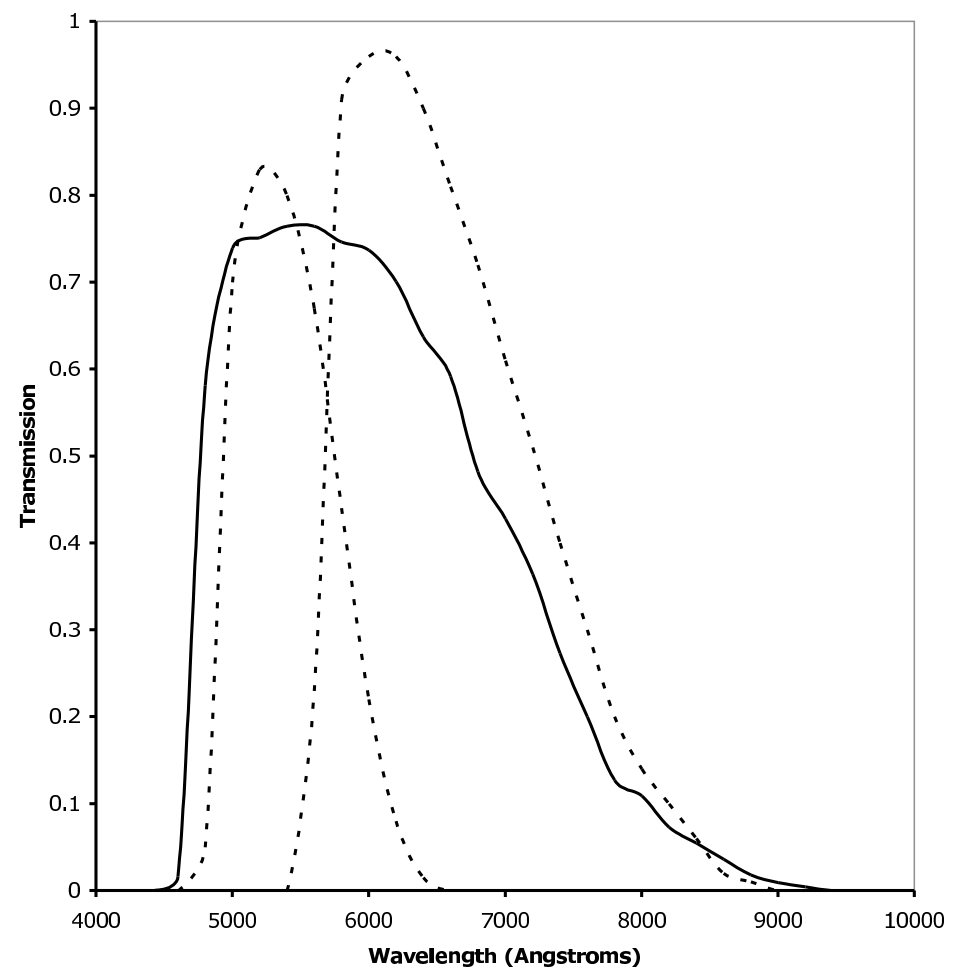

Figure 2. Filter Transmission curve (solid line) for RINGO custom filter (3mm GG475 + 2mm KG3) compared to Bessell $^{17} V$ and $R$ curves (dotted lines).

\subsection{Time Resolution}

As the polarization may be rapidly varying at early times, the instrument should be capable of measuring polarization on timescales of $\sim 10$ seconds.

\subsection{Wavelength range}

In order to derive the optimal wavelength range for the instrument, an analysis of the colours of early GRB light curves was undertaken. This showed they were on average red, with $B-V \sim V-R \sim 1$. A series of simple signal to noise ratio calculations showed that there was no advantage to observing in white light or blueward of the $V$ band in terms of final signal to noise ratio achieved, any additional signal gained being swamped by the additional sky. An additional concern was to avoid fringing on the CCD. Experience with RATCam shows that fringing becomes problematical in the $I$ band. A simple prescription for a $V+R$ filter was therefore derived. This consists of 3mm Schott GG475 cemented to $2 \mathrm{~mm}$ KG3. The resulting bandpass is shown in Figure 2.

\section{INSTRUMENT DESIGN}

\subsection{Prototyping}

A number of possible designs were explored in developing the instrument. The potentially rapid variability of the source meant that any designs that measured the polarization components sequentially (e.g. by rotating an analyzer to two positions in turn) were rejected. Some initial ideas along the lines of "conventional" imaging polarimeters, with a focal plane mask and a birefringent material and reimaging optics to produce "side by side" images of the ordinary and extra-ordinary rays were explored. However it proved difficult to meet the field of view requirement, especially as the source would be at an unknown position within that field of view. The eventual design arrived at was therefore based on the novel ring polarimeter concept which was recently explored 


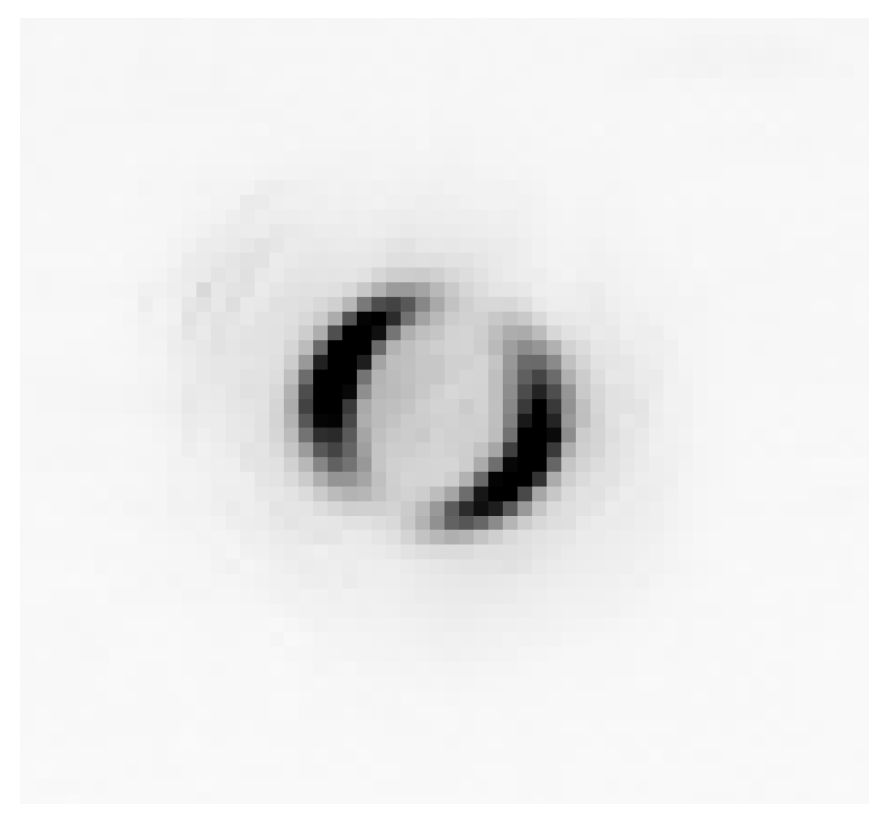

Figure 3. Image from RINGO prototype. The lab test source is strongly polarized.

by Clarke \& Neumayer ${ }^{18}$ based upon the principle proposed by Treanor. ${ }^{19}$ This uses a rotating Polaroid to modulate any polarized flux in the collected radiation and is followed by co-rotating deviating optics so that each star image is recorded on the CCD chip as a circular pattern. The polarization signal is therefore mapped out twice around the circumference of each recorded image. Reference ${ }^{18}$ provides more details of the advantages of such a device, including its potential for high precision measurements using CCD detectors (since it spreads the flux over many pixels around the ring, thereby reducing the normal saturation constraints on high precision CCD photometry). A potential disadvantage of the design is that in crowded fields the rings can overlap causing problems in extraction. However a short calculation showed that this was only a problem a low galactic latitudes, where GRB's are generally heavily extinguished by the galactic absorption and therefore unobservable anyway.

Following on from the experiments of Clarke \& Neumayer we initially built a small prototype of the design, using 1 inch optics held in a barrel and driven via a belt by a small AC synchronous motor taken from a recordplaying turntable. An amateur Starlight Express CCD was used as a detector. Both unpolarized and strongly polarized sources were used, and the resulting image for a $\sim 100 \%$ polarized source can be seen in Figure 3 .

\subsection{Design Optimizations}

The key parameters to be optimized in the design of the instrument are the speed of rotation of the polarizer/deflector combination and the geometry of the deflecting optics (which sets the ring diameter).

The speed of rotation has an impact on the polarimetric accuracy that can be obtained as any "non-integer" number of rotations with respect to the exposure time will impose a false signal on the ring profile. In theory one could synchronize the shutter and rotation, however in practice the simpler solution is simply to ensure the rotation speed is sufficiently fast that it does not impact the measured profile significantly. Some simple arithmetic shows that the percentage polarimetric error introduced by partial-rotations is approximated by

$$
\Delta P_{\text {rotation }} \sim=\frac{12000}{v \times t}
$$

where $v$ is the rotation speed in revolutions per minute and $t$ the exposure time in seconds.

The minimum ring diameter is set by the atmospheric seeing such that it does not cause flux to "spill" across the ring or between adjacent sections around the ring where independent measurements of the flux are made 


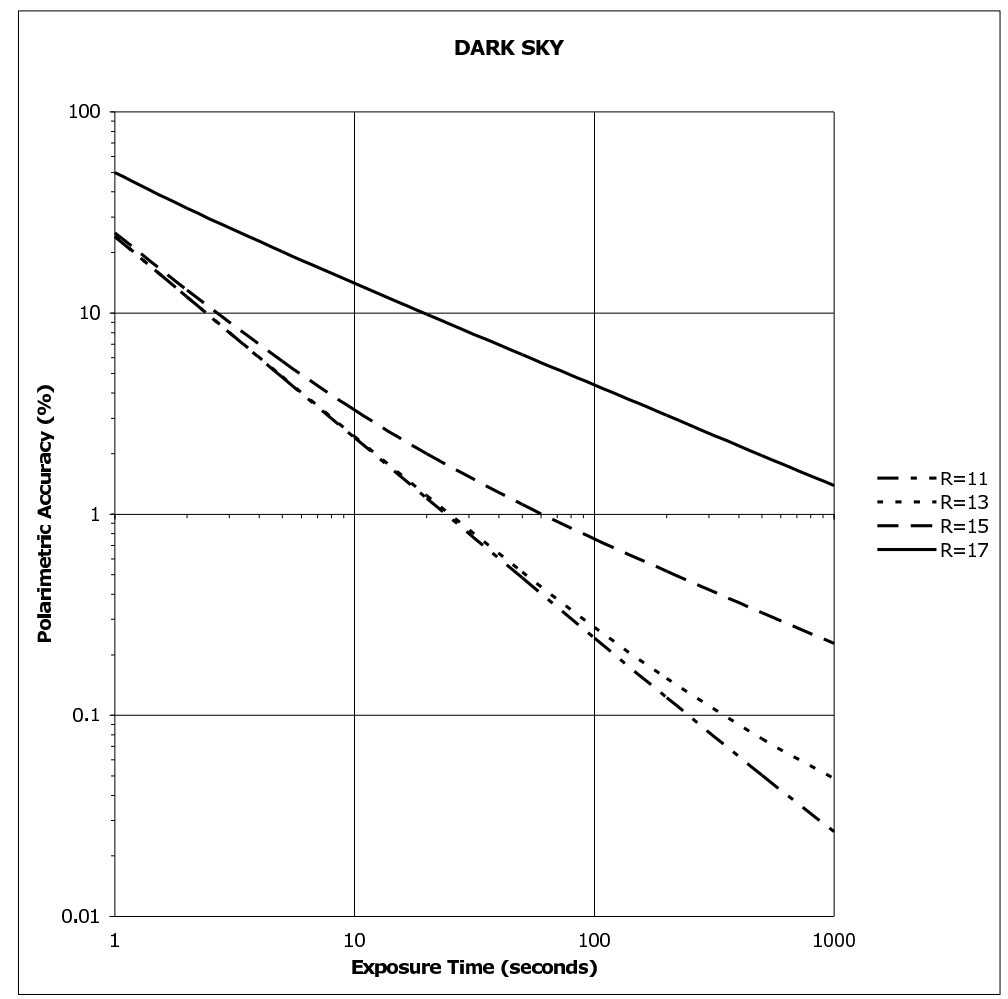

Figure 4. Results of simulations carried out during the design phase to determine the minimum ring rotation rate and optimal ring diameter. The curves shown are for the eventually adopted parameters of $500 \mathrm{rpm}$ and a diameter of 30 arcseconds. The results are for a dark sky background of $19.4 \mathrm{mag} /(\operatorname{arcsec})^{2}$

in the reduction process (Section 5). This must be traded off against the increased contribution of sky noise to the measurement that is made when the ring diameter (and hence the area of the ring) is made larger. The percentage polarimetric error due to this source can be approximated using simple photon counting statistics:

$$
\Delta P_{\text {photons }} \sim 100 \times \frac{\sqrt{N_{o b j}+N_{s k y}}}{N_{o b j}} .
$$

where $N_{o b j}$ and $N_{s k y}$ are the number of photons detected from the object and sky respectively. Assuming an instrumental zeropoint of 23.6 (based on the achieved zeropoint for direct CCD imaging with an $R$ band filter reduced by 1 magnitude to allow for the light loss through the polaroid and prism) the photon count from a source of magnitude $R_{o b j}$ is given by

$$
N_{o b j}=10^{\left(23.6-R_{o b j}\right) / 2.5} t,
$$

and the photon count from the sky (of brightness $R_{s k y}$ magnitudes $/(\operatorname{arcsec})^{2}$ is given by

$$
N_{s k y}=10^{\left(23.6-R_{s k y}\right) / 2.5} t * A
$$

where $A$ is the area of the ring. This can be calculated by simple geometry for a ring of width $w$ and diameter $D$ to be

$$
A=\pi\left(D+\frac{w}{2}\right)^{2}-\pi\left(D-\frac{w}{2}\right)^{2} .
$$

The final polarimetric error of the instrument can then be modelled by adding the rotation and photoncounting components of the error in quadrature. The results of doing this for stars of a range of different magnitudes with a rotator speed of $500 \mathrm{rpm}$ and a ring of diameter 30 arcseconds with a trace width 4 arcseconds are shown in Figures 4 and 5 for dark and bright sky conditions respectively. Note how for bright objects $(R<12)$ the achieved accuracy is dominated by the rotation speed, whereas for fainter objects photon statistics dominate. 


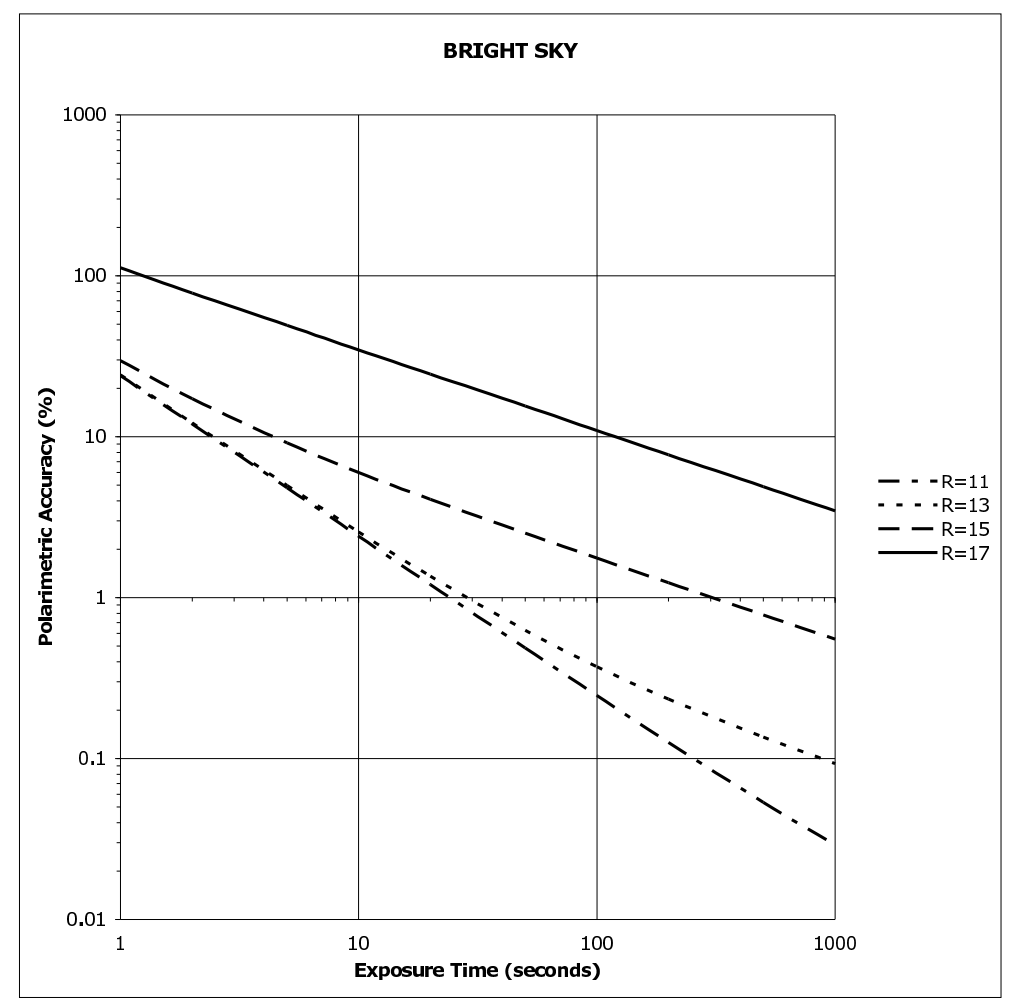

Figure 5. As Figure 4 but for a bright sky background of $17.4 \mathrm{mag} /(\operatorname{arcsec})^{2}$.

\subsection{Instrument construction}

For reasons of cost and speed, all optical components were chosen to be commercial off the shelf. The polarizer chosen was a Meadowlark Precision linear polarizer. This uses a dichroic polymer polarizer laminated between a BK7 substrate which is antireflection coated, giving a per-surface reflectance of $<0.5 \%$ at the operating wavelength range, and an overall throughput of $\sim 90 \%$. For the deviating element a 1.5 degree BK7 wedge prism was supplied by CVI Laser Optics. This was again anti-reflection coated.

An overall layout of the instrument is shows in Figure 6. The assembly was manufactured from a combination of steel and aluminium with Tufnol used where electrical isolation was necessary (e.g. between the CCD camera and instrument to allow control of Earth loops). The weight of the rotor is $\sim 1 \mathrm{~kg}$, and it is housed within two ball bearings. A rubber drive belt makes a friction contact with the rotor to provide a clutch action on start up. The use of a belt drive also isolates the rotor from any vibrations associated with the motor, which is compliantly mounted onto the instrument. The motor is a 30 watt brushless (to reduce electromagnetic interference during CCD readout) Creuzet high torque $24 \mathrm{~V}$ DC model. The motor rotation speed is $1500 \mathrm{rpm}$, which is reduced via the belt drive to 500rpm at the rotor. In operation the motor is left running continually (day and night) so that no delays for system spin-up are encountered on a GRB trigger.

The CCD camera is an the Apogee ALTA E42. This uses a back-illuminated, midband coated AIMO E2V CCD 42-40 and is thermoelectrically cooled to $-20^{\circ} \mathrm{C}$. The quantum efficiency in the operating wavelength range of the instrument is $>80 \%$ and the dark current $\sim 1$ electron/pixel/second at the operating temperature. The CCD is operated binned $2 \times 2$, giving a pixel scale of 0.27 arcseconds/pixel. In this mode the readout time is $<10$ seconds. The camera has a built in Ethernet interface, meaning that no special wiring or on mount IT or other electronic hardware provision needed to be made, the instrument simply plugging in to the telescope LAN. CCD readout and control software was developed built upon the Random Factory Linux device driver, implementing the LT standard generic command set and interface to the telescope robotic control system. ${ }^{2}$ 


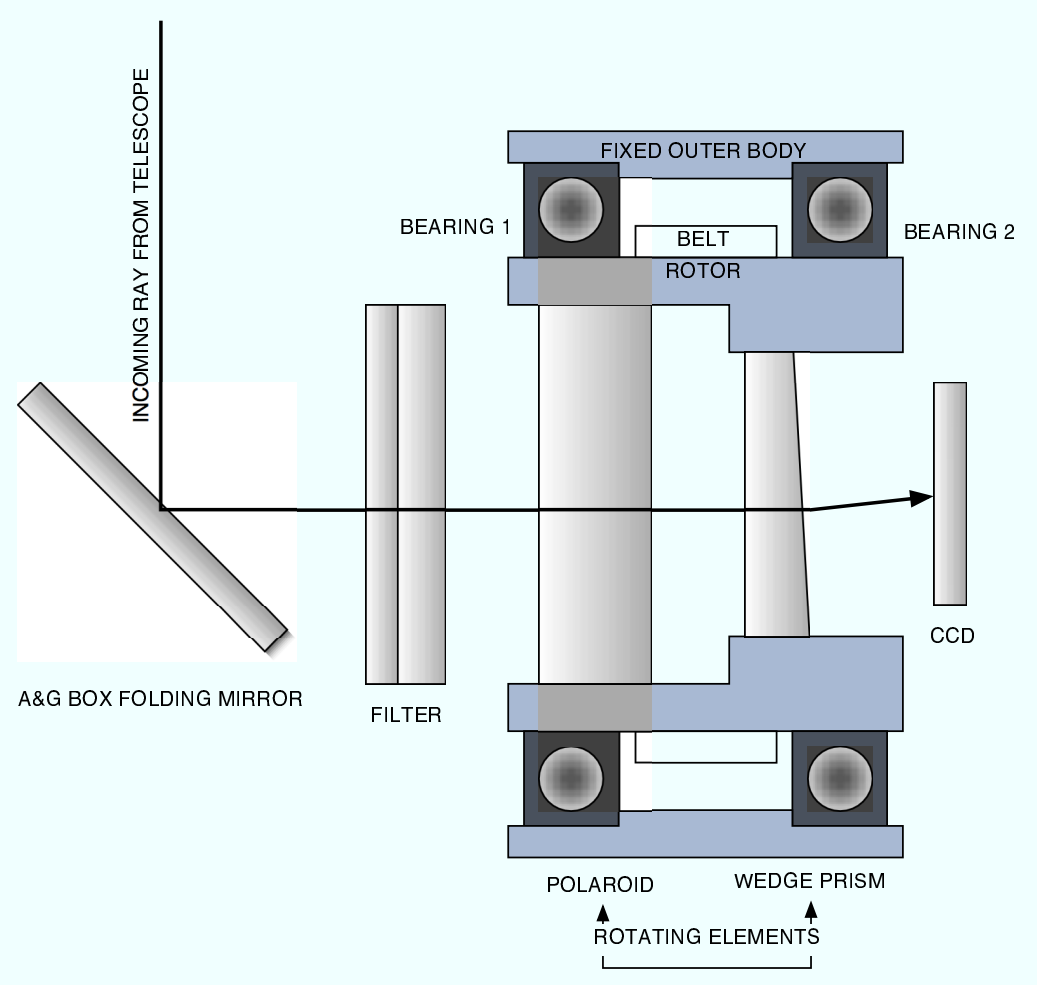

Figure 6. Basic layout of RINGO instrument (not to scale).

\section{DATA REDUCTION}

In theory the reduction of data from the instrument should be relatively straightforward. A trace of the image intensity around the ring is made, and that can be fitted to the equations given in Reference ${ }^{18}$ which allow the derivation of the Stokes parameters.

In practice the reduction of data is complicated by a number of factors. The beam is reflected off a 45 degree folding mirror before entering the instrument. This leads to a significant additional polarization signal being superimposed on the beam. Additionally there will also be some instrumental depolarization of the original source caused by the telescope and instrument optics. We note here that our final characterization of the instrument is still underway, and there are still some low level effects we do not understand in the data (for example there is apparently a $360^{\circ}$ modulation in the data as well as the expected $180^{\circ}$ one that is still under investigation). However we have found that a basic reduction and calibration of the data can be done using observations of polarization standards ${ }^{20}$ both of known zero and non-zero polarization.

The first stage of the data reduction is to extract ring profiles free of detector signature or any biases associated with the extraction process:

1. Subtract a dark frame of identical exposure length as the science frames from the original images.

2. Construct a master flat field by median filtering dark subtracted sky flats taken during twilight.

3. Divide the flat field into the science frame.

4. Identify the centre of the ring. Note this is crucial as misalignment will introduce a spurious signal into the trace around the ring. We have found that centre can be identified either using a Hough transform or cross-correlation techniques. 


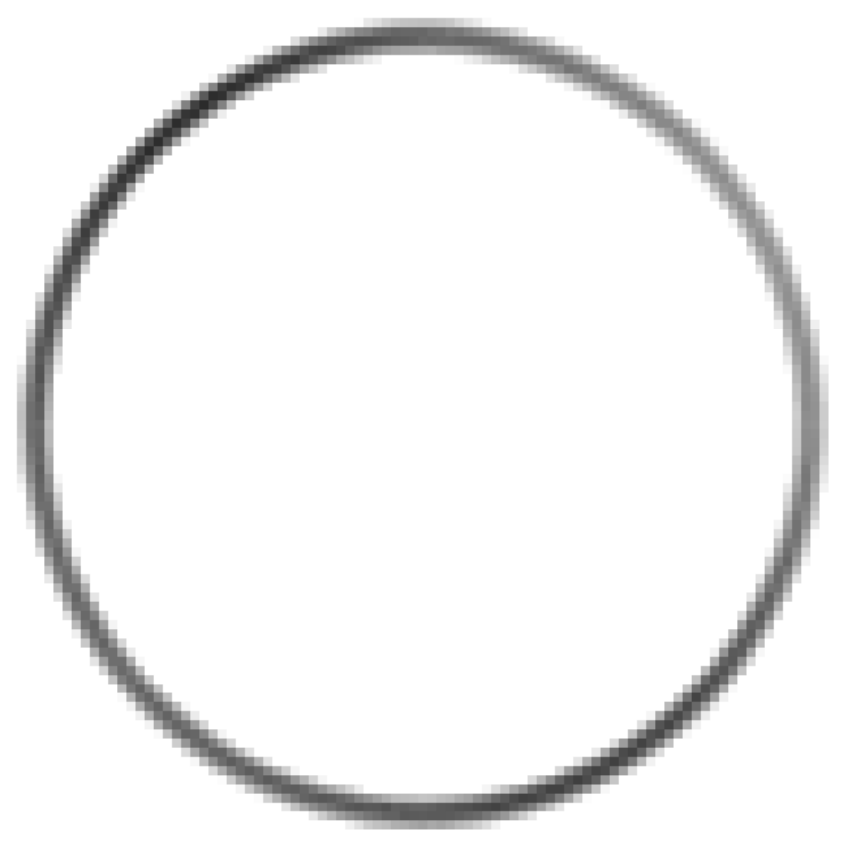

Figure 7. Image of the polarization standard BD +64 106 taken during on telescope commissioning.

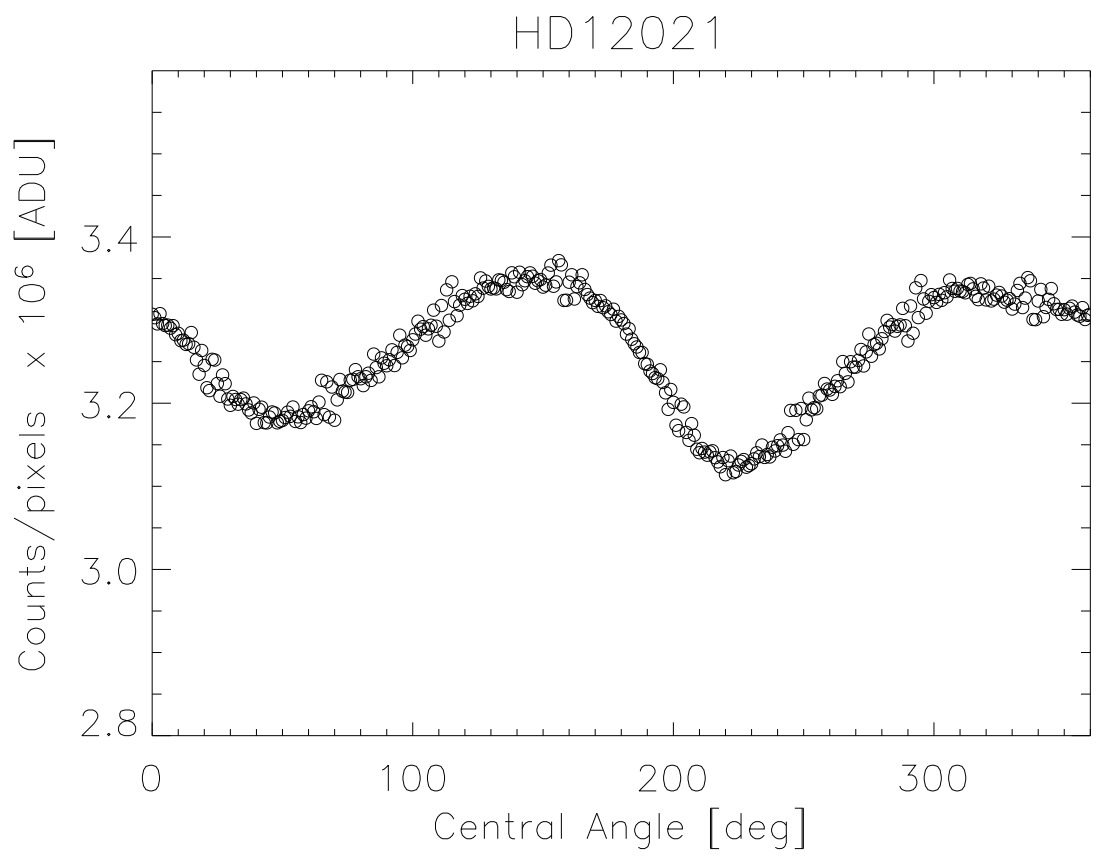

Figure 8. Sky subtracted intensity trace around the ring produced by the zero polarization standard HD12021. Angles are measured w.r.t North on the sky. The variation in signal is due to the instrumental polarization. 


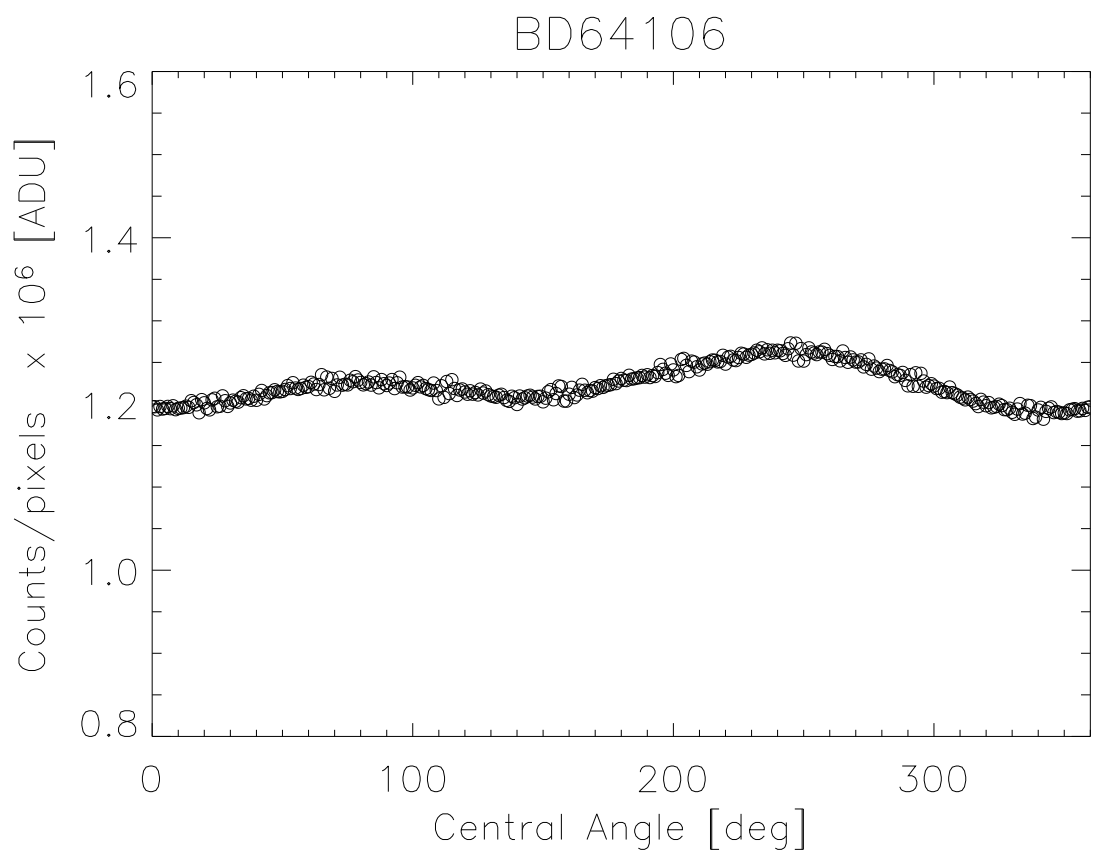

Figure 9. Trace around the polarization standard BD +64106 . The variation is small because the polarization signal happens to be out of phase with the instrumental signature in this case.

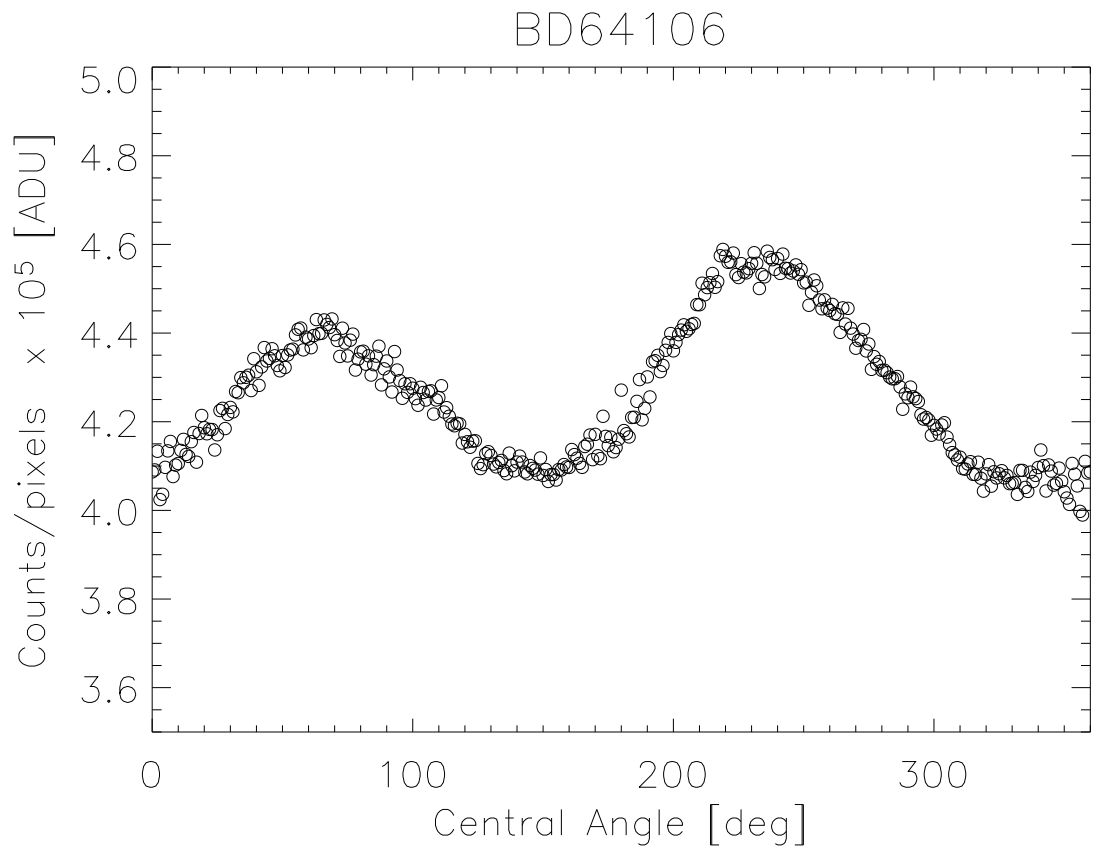

Figure 10. The polarization standard BD +64 106 with the instrumental polarization signature removed. 


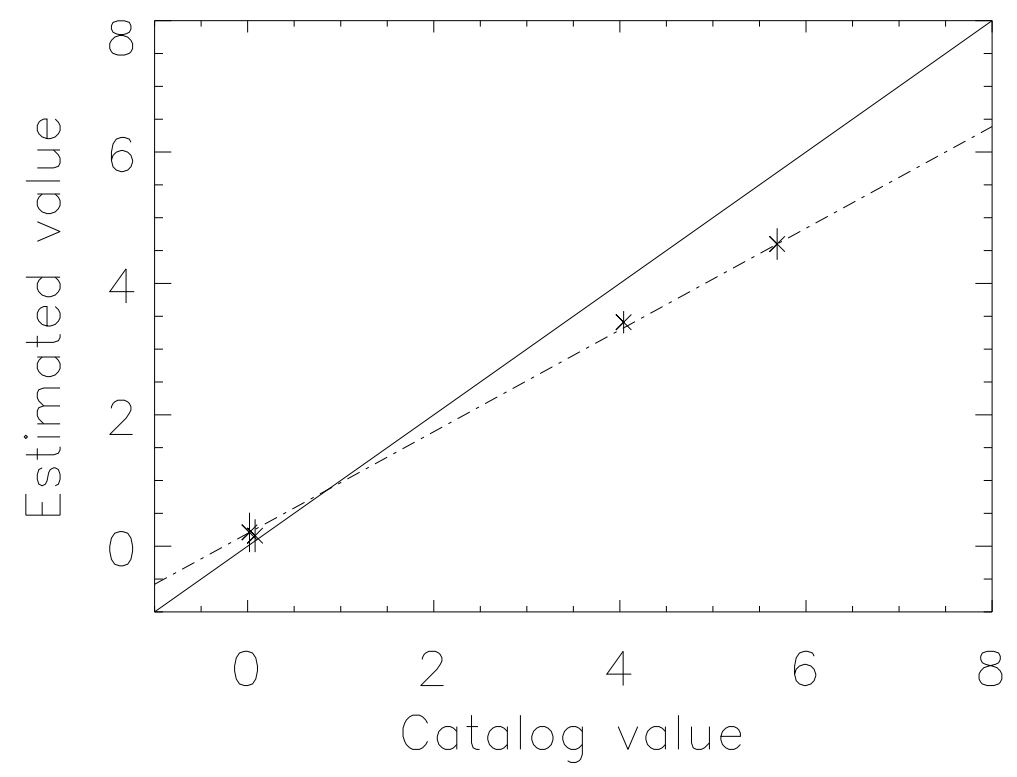

Figure 11. Measured versus catalogue polarization in $\%$ for a number of polarimetric standards.

5. Derive the flux in bins around the ring (typically $\sim 15^{\circ}$ ), optimizing the width of the bins so as to maximize signal-to-noise ratio while ensuring that any non-circular PSF does not introduce a spurious signal.

6. Derive a sky flux in bins outside the ring and scale according to the relative area of the sky and object rings, and then subtract from the object ring.

The above sequence is adopted both for the science and the polarimetric standard ${ }^{20}$ frames. The zeropolarization standard traces are then averaged to create a "template" trace. This aims to characterize the effects of instrumental polarization in much the same way as a flat-field does for instrumental photometric effects. However as the zero-polarization standards are not truly "zero polarization", suffering from interstellar and other astrophysical effects, an iterative technique is then required to derive a true zero polarization template. This can be created by dividing each of the nominally zero polarization standards by the template and fitting the polarization constrained by the knowledge of the actual polarization of the standard. The residuals from those fits averaged together over all the standards then will provide a better measure of the template. This procedure can then be repeated if necessary until a solution for template is arrived at. The final template should only contain the signature of the instrumental polarization. An example of a template constructed from only one object is shown in Figure 8.

In Figure 9 we show a trace around a sample non-zero polarimetric standard. Dividing the template from Figure 8 into the standard then yields Figure 10. This trace with the instrumental signature removed can then be fitted ${ }^{18}$ and the Stokes parameters derived, free from the effects of instrumental polarization. The derived polarization angle and degree of polarization can then be compared to the published standard values. This then allows the degree of instrumental depolarization to be derived (Figure 11) which can then be applied as a correction factor to the science frames. Our preliminary result for this correction gives:

$$
X=\frac{Y-0.1945}{0.7742}
$$

where $X$ is the real (catalogue) polarization and $Y$ is the measured (instrumental) value. 


\section{COMISSIONING RESULTS}

RINGO was commissioned on the Liverpool Telescope in November 2005. During commissioning a large number of polarimetric standard stars were observed, with repeated measurements made and testing done with the standard located in different parts of the field. An example image taken on the commissioning run can be seen in Figure 7. The instrument was left running on the telescope following commissioning, and is now in routine science use.

During commissioning tests of flat and dark frame stability and CCD linearity were carried out. Also tested were the saturation limits of the device (Table 1). The traces presented in Figures 8 - 11 are also from the commissioning run. As described above, our understanding of all the instrumental effects (including those contributed by the telescope) is not yet complete. However analysis of the repeated observations of standards shows that the degree of polarization can be accurately measured to $\sim 0.1 \%$, which is sufficient to make the determination of early time GRB polarization and its evolution possible.

Table 1. Saturation Limits

\begin{tabular}{|l|l|}
\hline Magnitude & Maximum Exposure Time \\
\hline 6 & 1 \\
\hline 7 & 2 \\
\hline 8 & 5 \\
\hline 9 & 10 \\
\hline 10 & 30 \\
\hline 11 & 60 \\
\hline 12 & 120 \\
\hline
\end{tabular}

\section{CONCLUSION}

The RINGO instrument was developed and deployed in under 6 months and for a budget of $£ 30,000(\sim \$ 50,000)$, the majority of which was the cost of the CCD camera. Its novel design gives it a wide field of view and the fast response necessary for measuring the polarization of gamma ray burst optical transients. Commissioning tests have shown that it can make repeatable measurements to a precision of $0.1 \%$. It is now in routine operation on the Liverpool Telescope for Gamma Ray Burst followup and other science programmes requiring regular monitoring of variable polarization sources.

\section{ACKNOWLEDGMENTS}

The Liverpool Telescope is located at the Observatorio del Roque do Los Muchachos, La Palma, Canary Islands, Spain. It was designed and built by Telescope Technologies Ltd and is owned and operated by Liverpool John Moores University with financial support from the UK PPARC.

The hardware costs of RINGO were supported using funding from the UK HEFCE SRIF2 project.

\section{REFERENCES}

1. I. A. Steele, R. J. Smith, P. C. Rees, I. P. Baker, S. D. Bates, M. F. Bode, M. K. Bowman, D. Carter, J. Etherton, M. J. Ford, S. N. Fraser, A. Gomboc, R. D. J. Lett, A. G. Mansfield, J. M. Marchant, G. A. Medrano-Cerda, C. J. Mottram, D. Raback, A. B. Scott, M. D. Tomlinson, and R. Zamanov, "The Liverpool Telescope: performance and first results," in Proceedings of the SPIE, Volume 5489, pp. 679-692 (2004)., J. M. Oschmann, ed., pp. 679-692, Oct. 2004. 
2. C. J. Mottram, I. A. Steele, and L. Morales, "Design of low cost and reliable instrumentation for robotic telescopes," in Ground-based Instrumentation for Astronomy. Edited by Alan F. M. Moorwood and Iye Masanori. Proceedings of the SPIE, Volume 5492, pp. 677-688 (2004)., A. F. M. Moorwood and M. Iye, eds., pp. 677-688, Sept. 2004.

3. C. Guidorzi, A. Monfardini, A. Gomboc, C. J. Mottram, C. G. Mundell, I. A. Steele, D. Carter, M. F. Bode, R. J. Smith, S. N. Fraser, M. J. Burgdorf, and A. M. Newsam, "The Automatic Real-Time Gamma-Ray Burst Pipeline of the 2 m Liverpool Telescope," PASP 118, pp. 288-296, Feb. 2006.

4. C. Guidorzi, A. Monfardini, A. Gomboc, C. G. Mundell, I. A. Steele, D. Carter, M. F. Bode, R. J. Smith, C. J. Mottram, M. J. Burgdorf, N. R. Tanvir, N. Masetti, and E. Pian, "The Early Multicolor Afterglow of GRB 050502a: Possible Evidence for a Uniform Medium with Density Clumps," ApJL 630, pp. L121-L124, Sept. 2005.

5. A. Gomboc, M. F. Bode, D. Carter, C. Guidorzi, A. Monfardini, C. G. Mundell, A. M. Newsam, R. J. Smith, I. A. Steele, and J. Meaburn, "Rapid GRB Follow-up with the 2-m Robotic Liverpool Telescope," in AIP Conf. Proc. 797: Interacting Binaries: Accretion, Evolution, and Outcomes, L. Burderi, L. A. Antonelli, F. D'Antona, T. di Salvo, G. L. Israel, L. Piersanti, A. Tornambè, and O. Straniero, eds., pp. 181-186, Oct. 2005.

6. E. Costa, F. Frontera, J. Heise, M. Feroci, J. in 't Zand, F. Fiore, M. N. Cinti, D. dal Fiume, L. Nicastro, M. Orlandini, E. Palazzi, M. Rapisarda, G. Zavattini, R. Jager, A. Parmar, A. Owens, S. Molendi, G. Cusumano, M. C. Maccarone, S. Giarrusso, A. Coletta, L. A. Antonelli, P. Giommi, J. M. Muller, L. Piro, and R. C. Butler, "Discovery of an X-ray afterglow associated with the gamma-ray burst of 28 February 1997.," Nature 387, pp. 783-785, June 1997.

7. J. van Paradijs, P. J. Groot, T. Galama, C. Kouveliotou, R. G. Strom, J. Telting, R. G. M. Rutten, G. J. Fishman, C. A. Meegan, M. Pettini, N. Tanvir, J. Bloom, H. Pedersen, H. U. Nordgaard-Nielsen, M. LindenVornle, J. Melnick, G. van der Steene, M. Bremer, R. Naber, J. Heise, J. in 't Zand, E. Costa, M. Feroci, L. Piro, F. Frontera, G. Zavattini, L. Nicastro, E. Palazzi, K. Bennet, L. Hanlon, and A. Parmar, "Transient optical emission from the error box of the gamma-ray burst of 28 February 1997.," Nature 386, pp. 686-689, 1997.

8. T. Piran, "The physics of gamma-ray bursts," Reviews of Modern Physics 76, pp. 1143-1210, Jan. 2005.

9. S. Covino, D. Lazzati, G. Ghisellini, P. Saracco, S. Campana, G. Chincarini, S. di Serego, A. Cimatti, L. Vanzi, L. Pasquini, F. Haardt, G. L. Israel, L. Stella, and M. Vietri, "GRB 990510: linearly polarized radiation from a fireball," $A \& A$ A 348, pp. L1-L4, Aug. 1999.

10. R. A. M. J. Wijers, P. M. Vreeswijk, T. J. Galama, E. Rol, J. van Paradijs, C. Kouveliotou, T. Giblin, N. Masetti, E. Palazzi, E. Pian, F. Frontera, L. Nicastro, R. Falomo, P. Soffitta, and L. Piro, "Detection of Polarization in the Afterglow of GRB 990510 with the ESO Very Large Telescope," ApJL 523, pp. L33-L36, Sept. 1999.

11. J. Greiner, S. Klose, K. Reinsch, H. Martin Schmid, R. Sari, D. H. Hartmann, C. Kouveliotou, A. Rau, E. Palazzi, C. Straubmeier, B. Stecklum, S. Zharikov, G. Tovmassian, O. Bärnbantner, C. Ries, E. Jehin, A. Henden, A. A. Kaas, T. Grav, J. Hjorth, H. Pedersen, R. A. M. J. Wijers, A. Kaufer, H.-S. Park, G. Williams, and O. Reimer, "Evolution of the polarization of the optical afterglow of the $\gamma$-ray burst GRB030329," Nature 426, pp. 157-159, Nov. 2003.

12. G. Björnsson, E. H. Gudmundsson, and G. Jóhannesson, "Energy Injection Episodes in Gamma-Ray Bursts: The Light Curves and Polarization Properties of GRB 021004," ApJL 615, pp. L77-L80, Nov. 2004.

13. S. Covino, G. Ghisellini, D. Lazzati, and D. Malesani, "Polarization of Gamma-Ray Burst Optical and Near-Infrared Afterglows," in Astronomical Society of the Pacific Conference Series, M. Feroci, F. Frontera, N. Masetti, and L. Piro, eds., pp. 169-+, Apr. 2004.

14. J. Granot and A. Königl, "Linear Polarization in Gamma-Ray Bursts: The Case for an Ordered Magnetic Field," ApJL 594, pp. L83-L87, Sept. 2003.

15. D. Lazzati, S. Covino, J. Gorosabel, E. Rossi, G. Ghisellini, E. Rol, J. M. Castro Cerón, A. J. Castro-Tirado, M. Della Valle, S. di Serego Alighieri, A. S. Fruchter, J. P. U. Fynbo, P. Goldoni, J. Hjorth, G. L. Israel, L. Kaper, N. Kawai, E. Le Floc'h, D. Malesani, N. Masetti, P. Mazzali, F. Mirabel, P. Moller, S. Ortolani, E. Palazzi, E. Pian, J. Rhoads, G. Ricker, J. D. Salmonson, L. Stella, G. Tagliaferri, N. Tanvir, E. van den 
Heuvel, R. A. M. J. Wijers, and F. M. Zerbi, "On the jet structure and magnetic field configuration of GRB 020813," A\&SA 422, pp. 121-128, July 2004.

16. E. M. Rossi, D. Lazzati, J. D. Salmonson, and G. Ghisellini, "The polarization of afterglow emission reveals $\gamma$-ray bursts jet structure," MNRAS 354, pp. 86-100, Oct. 2004.

17. M. S. Bessell, "UBVRI passbands," PASP 102, pp. 1181-1199, Oct. 1990.

18. D. Clarke and D. Neumayer, "Experiments with a novel CCD stellar polarimeter," $A \mathscr{E} A$ 383, pp. 360-366, Jan. 2002.

19. P. F. Treanor, "A new technique for stellar polarimetry," MNRAS 138, pp. 325-+, 1968.

20. G. D. Schmidt, R. Elston, and O. L. Lupie, "The Hubble Space Telescope Northern-Hemisphere grid of stellar polarimetric standards," AJ 104, pp. 1563-1567, Oct. 1992. 